\title{
Statistical analysis on a large time-sharing system
}

\author{
WILLIAM A. LEAF† \\ Carnegie-Mellon University, Pittsburgh, Pennsylvania 15213
}

\begin{abstract}
A set of statistical routines for strictly conversational use is discussed. The routines are being developed at Carnegie-Mellon University, where the main time-sharing system is TSS installed on an IBM $360 / 67$, and they exist at several levels.
\end{abstract}

Statistical routines and "packages" have long been found at moderately large and very large computer installations. Traditionally they have been geared to batch operation. To do a particular analysis, one prepared a deck of control cards, program, parameter choices, and data and fed the deck to the machine. Recently it has become possible to keep programs and/or data accessible on disk storage devices, so that one might need to submit a deck consisting only of control cards.

To a large extent, however, time-sharing monitors are now controlling the large university computers that have major statistical packages. Those packages have been converted to run under the new monitors, but the routines fail to take advantage of the interactive opportunity in time-sharing. At best, one can enter parameters and data from a terminal while the program runs, just as if the terminal input was a prepunched deck of cards being read one at a time.

Two main features need to be added to such routines to make them truly conversational: verbal prompts to the user whenever input is needed (e.g., ENTER THEORETICAL PROPORTION:) and error correcting facilities which ask the user's help in recovering from invalid input (one way is simply rerequesting data, e.g., PROPORTION MUST BE BETWEEN $\emptyset$ and 1 ; REENTER:).

Additional changes to tailor routines to user thinking are useful but the changes can go on forever. One kind relevant to time-sharing is postponing requests for option choices until they are appropriate-asking for information at the time it is needed in the analysis, for example, or delaying option requests until intermediate output relevant to the option choice has been printed.

A set of statistical routines for strictly conversational use is being developed at Carnegie-Mellon University, where the main time-sharing system is TSS installed on an IBM 360/67. The routines exist at several levels. Most coding has taken place at the "statistical tool

\footnotetext{
*This work was in part supported by National Institute of Mental Health Research Grant MH-07722 at Carnegie-Mellon University.

†Requests for reprints should be sent to William A. Leaf, Department of Psychology, Carnegie-Mellon University, Schenley Park, Pittsburgh, Pennsylvania 15213.
}

subroutine" level: random number generators, significance tests, generalized subscripting routines, and generalized vector and matrix operations. A second level involves simplified conversational $\mathrm{I} / \mathrm{O}$ for FORTRAN programs, based on completely free-format input and the notion of a dialogue between program and user, in which the program is either telling the user something or asking him for some choice or information. The third level, the least active thus far, has been main statistical routines which are directly visible to the user and which do his analyses at his bidding. Only four programs exist at this level at this time: a generalized binomial (sign test) program, a chi-squared program, a student's t test program, and a factorial analysis of variance program. The last is the only one used extensively so far and will be used as the illustration in the discussion below.

\section{USERS}

Several assumptions are made by any programmer about the class of user for whom his program is intended. The accuracy of these assumptions is most important in a time-sharing environment, in which the user interacts alone in a real-time situation via a terminal. Our assumptions are general: The user need have only minimal familiarity with TSS-enough knowledge to access the system and type two or three cookbook commands. But he needs to know what programs are available; a list is adequa ${ }^{+} e$ here. He may be a student in a beginning statistics course, although these routines are not intended to teach statistics, or he may be an advanced student, sophisticated researcher with data to analyze, or faculty member. He may have sophisticated knowledge of statistics or minimal knowledge. He may intend only a basic analysis or want a complicated, detailed analysis.

\section{ANALYSIS OF VARIANCE PROGRAM}

The initial version of this program was written by Glenn Lea; frequent revisions have been made by this author. The program allows factorial designs of up to 12,000 data points and about 7000 separate cells and up to 10 factors of as many levels per factor as desired. It 


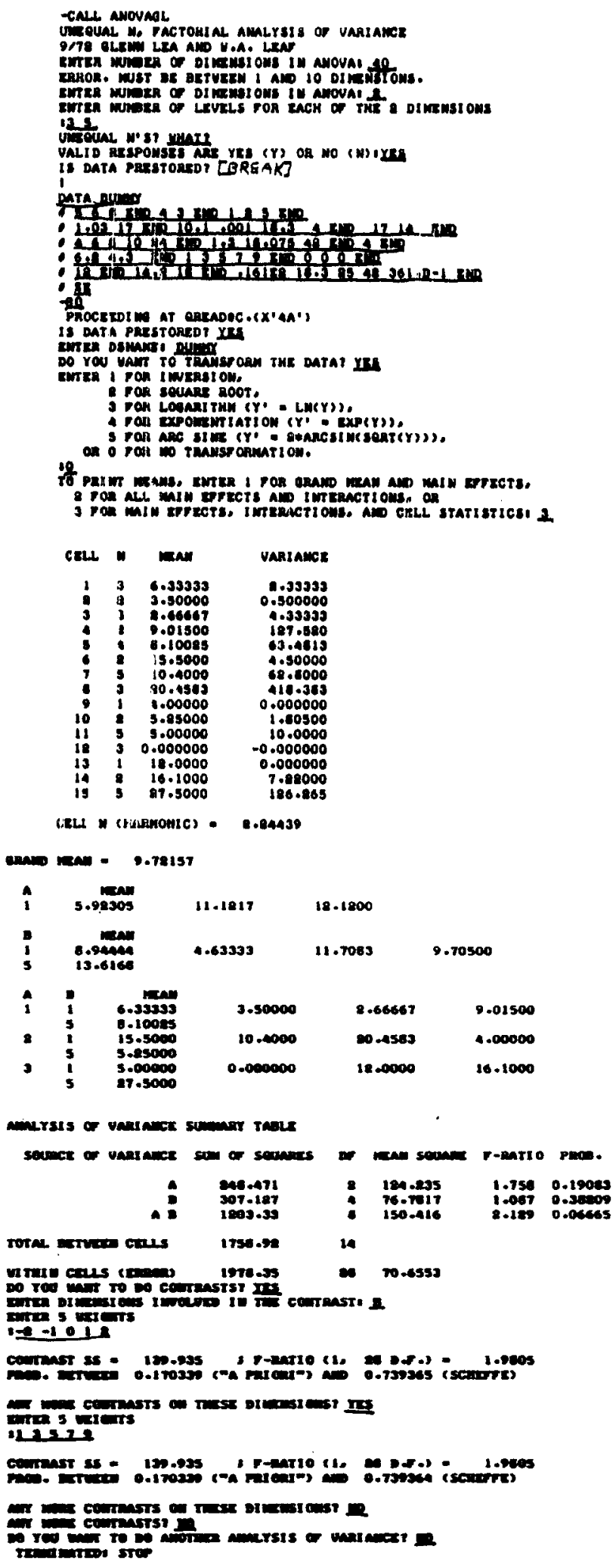

Fig. 1. Sample conversational analysis of variance.

includes such options as unequal cell frequencies, several data transformations, and flexible trend analysis powers. It reports means if desired and a complete analysis of variance summary table, including $F$ ratios and probability levels (assuming a fixed-effects model). For contrasts, the program reports a probability range, from the liberal "a priori" criterion to the very conservative Scheffé criterion. (In analysis of variance, contrast $F$ ratios may be interpreted in a variety of ways. For the expert, the reported probability range may have all the information he needs for his interpretation; for the novice, the reported range should serve as a clue that a straightforward interpretation may be invalid.) The program tends to read all information from the user's terminal. Large data sets, though, would require much time to enter and would frequently contain errors; they may be stored separately on disk and read during program execution.

\section{PROGRAM AND USER}

Figure 1 contains a record of a sample analysis of variance run. User responses are underlined. Included in the run are several user errors to illustrate the kinds of corrective measures the program will take. Also included is an example of another useful conversational facility: the ability to stop processing in one mode, do something else, and resume in the first mode at will. (The DATA DUMMY command creates a data set ["DUMMY"] on disk storage which, in this case, contains the data for the analysis. Each "\#" is a prompt for a new line, and "\%E" is the user response which indicates the data set is complete. GO returns to the analysis of variance program.)

The options available to the user are presented each time he runs the program. Hopefully, the comments are complete enough to inform the novice of his choices and requirements and brief enough not to drive the expert to distraction. There is only one version of the program, so that novices and experts must share the same comments. It is feasible to make more or less complete comments as a function of the user's competence, but so far the single version has proved adequate.

Hopefully, too, the comments are an adequate program description. One design goal for these main statistical routines is their completeness: The user should be able to properly use the routines with only the terminal in front of him-no external documentation and no huge store of memorized rules. One bit of evidence for our success is that, for a variety of reasons, no written document exists to describe this analysis of variance program; users have succeeded quite well without it.

\section{USE TO DATE}

Each program reports each time it is used, by whom, and when. Thus, it is possible to compile accurate use figures per program. Between September 1972, when the programs were first made available, and October 25, 1973, the analysis of variance program has been used 


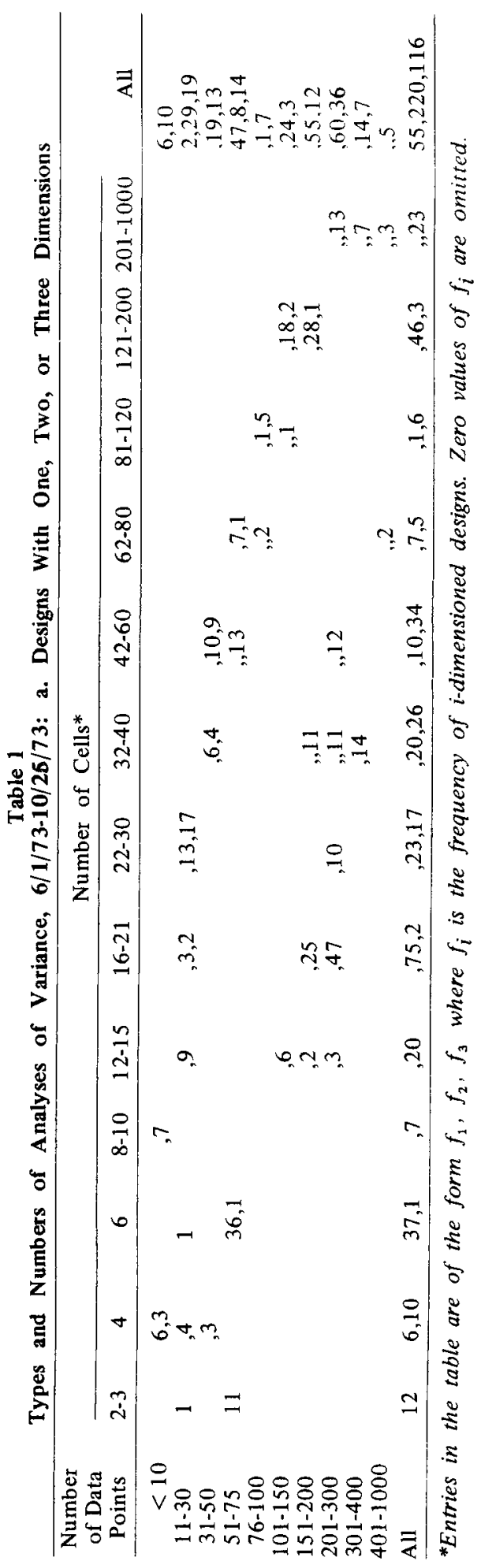

about 600 times. Most of these were for research analyses by graduate students and faculty. The other programs were used about 50 times each.

For the last 5 months, the analysis of variance program has also reported the types of analyses done-the number of dimensions in the design, the number of data cells and total data points, and the type
Table 1

b. Designs With Four or Five Dimensions

\begin{tabular}{|c|c|c|c|c|c|c|c|c|}
\hline \multirow{2}{*}{$\begin{array}{l}\text { Number } \\
\text { of Dimen- } \\
\text { sions }\end{array}$} & \multicolumn{8}{|c|}{ Number of Cells and Data Points } \\
\hline & $\begin{array}{l}31- \\
50\end{array}$ & $\begin{array}{r}76- \\
120\end{array}$ & $\begin{array}{l}121- \\
200\end{array}$ & $\begin{array}{l}201- \\
300\end{array}$ & 704 & 1344 & 1728 & All \\
\hline 4 & 1 & 1 & 13 & & 2 & 1 & 1 & 19 \\
\hline 5 & 1 & & 7 & 1 & & & & 9 \\
\hline
\end{tabular}

tAll analyses done during this period with four or five dimensions had exactly one score per cell.

of data transfomation used, if any. These data are summarized in Table 1. In general, the typical design has used two dimensions, 20 cells, and about 200 data points. The arc-sine transformation has been used most frequently, but only in about $14 \%$ of the cases.

\section{TECHNICAL DETAILS AND ASSESSMENT}

The main analysis of variance program is written in FORTRAN IV and includes about 400 statements. Most of these are involved in conversational $\mathrm{I} / \mathrm{O}$; if a single formatted READ statement was used, for example, with no error checking, about 200-300 statements could be eliminated.

Even with this large program, most work is done in subroutines. The complete analysis is done in a 60 -statement subroutine, and most of the $\mathrm{I} / \mathrm{O}$ work is done in a large series of other subroutines. These are necessary at this stage because FORTRAN has $1 / 0$ facilities that are limited, inflexible, and awkward for people. Conversational facilities, especially completely free-format input, should be present in any general purpose languages used in time-sharing environments.

The TSS configuration at Carnegie-Mellon University allows programs with huge storage requirements, so programs of this type are feasible here. They are less feasible elsewhere.

Conversational features which anticipate user errors and attempt reasonable corrections are expensive, in programmer effort and in program size. User acceptance has been good, however. The standard user errors with batch programs, such as data incorrectly aligned and options invalidly specified, seem not to occur (or are recognized immediately by the user and corrected on the spot). Users do not need to know FORTRAN or any of its idiosyncrasies and need only minimal knowledge of TSS, the host time-sharing system. They are able to concentrate on their data analyses and to use the flexibility of the programs almost immediately.

\section{FUTURE PLANS}

Other main user programs will be added as time and user interest demand. The programs that exist now will be opened to the entire campus this fall and their use by the wider community will be assessed. More complete information will be reported by the analysis of variance 
program, including a tally of user errors by type.

In a sense, the evolution of these routines is coming full circle. Because of the pricing structure on TSS, which favors batch operations, and because of the large volume of conversational use during prime hours, several users have begun using the analysis of variance program in batch operations. The commands are arbitrary and meaningless in a batch mode without the attendant comments $(2 / 3,5 / Y$, etc.). Thus, a "final" version of the program, to be installed in late 1973 , includes the option of a batch command set which is meaningful by itself. The input for the analysis shown in Fig. 1, for example, would be:

NDIM 2 LEVELS 35 DATA

[data for 15 cells as organized in "DUMMY" in Fig. 1.]

CONTRAST B $-2-1012$ STOP

\title{
An interactive computer program for tailored testing based on the one-parameter logistic model
}

\author{
MARK D. RECKASE* \\ University of Missouri, Columbia, Missouri 65201
}

\begin{abstract}
A program to implement tailored testing using the Rasch one-parameter logistic model is described and the problems encountered in its writing are discussed.
\end{abstract}

The concept of tailored testing (the administration to an individual of a specific set of items selected as appropriate) has been given considerable attention in the last 10 years in an attempt to overcome the problems inherent in the traditional objective testing situation. These problems concern time pressures on the individual, inefficient use of examinee time, inefficient use of item pools, lack of feedback, lack of objective success criteria, and many others. Reviews of the literature by Weiss and Betz (1973) and Cleary, Linn, and Rock (1968) discuss these problems in detail, so they will not be discussed here. The purpose of this paper is to present a computer program as one of the possible ways of solving these problems and to discuss the decisions that needed to be made in writing this program in the areas of (1) item selection, (2) scoring, (3) classification criteria, and (4) dimensionality of the item pool.

The theoretical base for the program is the one-parameter logistic model, commonly called the Rasch model (Rasch, 1960). This model relates the probability that individuals will answer item i correctly with a particular function of an individual's ability parameter and an item's easiness parameter. The relationship is given by the following formula:

$$
P\left\{X_{s i}\right\}=\frac{\left(A_{s} E_{i}\right)^{X_{s i}}}{1+A_{s} E_{i}}, X_{s i}=0,1
$$

where $\mathrm{X}_{\mathrm{si}}=0$ if the item was answered incorrectly and 1

* Requests for reprints should be sent to Mark D. Reckase, 8 Hill Hall, University of Missouri, Columbia, Missouri 65201. if it was answered correctly, $A_{\mathbf{s}}$ is a parameter indicating person s's ability, and $E_{i}$ is the easiness of item $i$. This model is a special case of the general three-parameter logistic model (Birnbaum, 1968):

$$
P\left\{X_{s i}=1\right\}=c_{i}+\frac{\left(1-c_{i}\right) e^{a_{i}\left(\theta_{s}-b_{i j}\right)}}{1+e^{a_{i}\left(\theta_{s}-b_{i}\right)}}
$$

when $A_{s}=e^{\theta} s, E_{i}=e^{-b_{i}}, a_{i}=1$, and $c_{i}=0$. In the three-parameter model, $c_{i}$ is the guessing parameter for the item, $a_{\mathbf{i}}$ is the discrimination parameter, $b_{\mathbf{i}}$ is the difficulty parameter, and $\theta_{\mathrm{s}}$ is the ability parameter for individual s.

The simpler Rasch model has been chosen for this program for several reasons. First, using the model, the ability parameters and item easiness parameters can be estimated independently (Rasch, 1960). This fact allows ability to be estimated on the same scale regardless of the set of items that is administered and allows items to be calibrated on groups at any ability level. The result of these estimation procedures is that item calibration can be performed conveniently on whatever groups happen to be available.

The second reason for using the one-parameter model is that efficient estimation procedures are available for estimating the parameters (Wright \& Panchepakesan, 1969). The estimation procedures for the three-parameter model have so far been found to be much too lengthy for extensive use (Lord, 1968).

Finally, the one-parameter model is a special case of the exponential family of distributions which has the properties that the number of correct responses obtained 